nationalistic manoeuvring. The astronomical community today is sensitive to this issue; for example, large river channels are now named using the word for Mars or star in various languages, and small craters are named after towns and villages from across the globe.

Professional astronomers have criticized Lowell for his cultivation of the media. Some planetary scientists today are similarly uneasy about the part that publicity plays in the Mars exploration programme. Discoveries and their implications are kept confidential and announced with great fanfare at press conferences before being presented and challenged at scientific meetings. As a consequence, a more sensational interpretation of a newly discovered feature can get the most attention, irrespective of its merit.

After the Viking Mars landers failed to detect life in the late 1970s, geneticist Norman
Horowitz cautioned that the seductive idea that life could have started on Mars means we should take care in interpreting new findings and presenting them to the public. Lane's book reminds us that is still good advice.

Michael Carr is a planetary scientist with the US Geological Survey, Menlo Park, California, USA.

e-mail:carr@usgs.gov

\title{
The brain in a nutshell
}

\section{Martin Kemp explains the resonances of Pascale Pollier's autopsy-inspired sculpture.}

$\mathrm{M}$ edicine has long used visual representation in ambitious ways. This is particularly true if we include the illustrated herbals inspired by the great five-volume encyclopaedia by Dioscorides (AD 40-90). Because art has traditionally centred on issues of human existence, medicine has also inspired many artists.

Recent works based on medical themes have tended to use metaphor and allusion rather than direct illustration. A striking example is provided by Belgian biomedical artist and poet Pascale Pollier, in a sculpture currently on show in the exhibition Picturing Science at the Riverside Gallery in Richmond, near London.

Her intense piece is enigmatically entitled Autopsy in a Nutshell. A bell jar, into which two coils of wire enter, contains a magnifying glass, two light-emitting diodes and a jointed stand with two sprung clamps. The beaks of the clamps grip a small model of the human brain and half of a walnut shell, the inside of which has been minutely remodelled to match the inside of a cranium.

As the title suggests, it was inspired by Pollier's witnessing of an autopsy. The first version of the piece was commissioned by Belgian learning expert Bernard Lernout, a great aficionado of Leonardo da Vinci and a fan of Michael Gelb's historically eccentric but creatively ingenious book, How to Think Like Leonardo da Vinci (Delacorte Press, 1998). Lernout directed Pollier to Gelb's seven Leonardesque "principles": curiosity (curiosità), demonstration (dimostrazione), sensation (sensazione), smokiness or ambiguity (sfumato, a layered paint effect), art-science (arte/scienza), embodiment (corporalità) and the connections between things (connessione).

Pollier picked up on three of these: demonstration, defined by Gelb as "learning
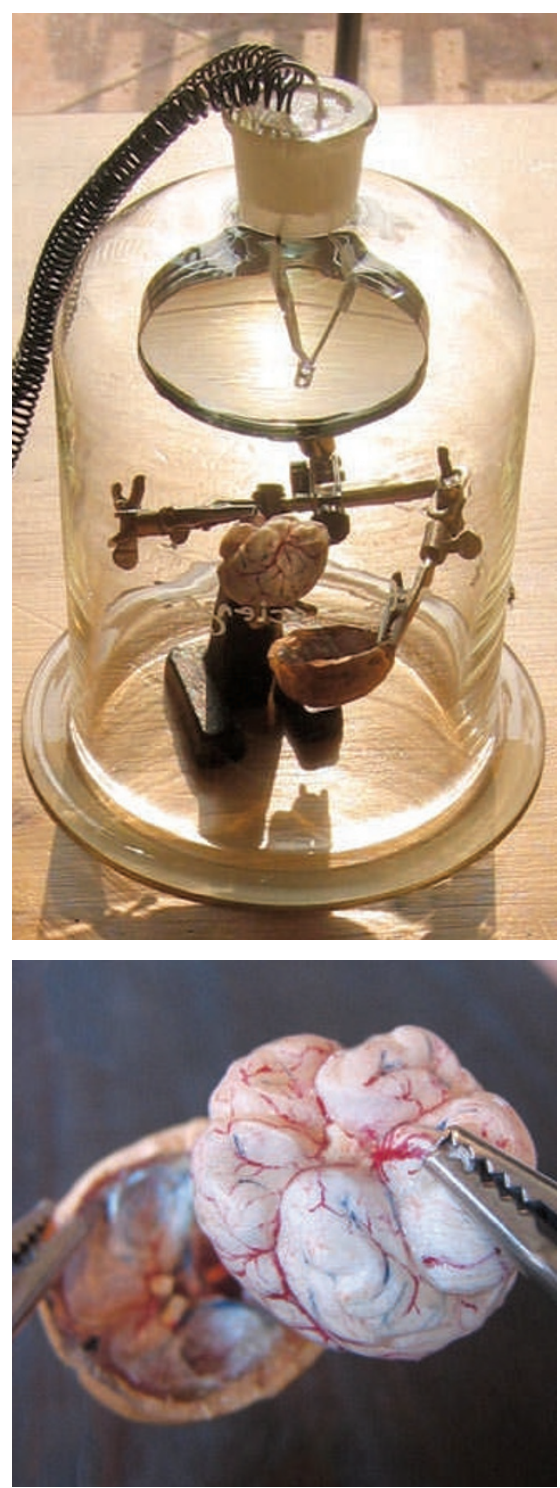

Autopsy in a Nutshell (2006) exploits more than the visual similarity between the brain and a walnut, as revealed on closer inspection (bottom).
Picturing Science from experience"; artRiverside Gallery, science, as balancing Richmond, UK. the properties of the Until 26 February two sides of the brain; 2011. and connection, as the need to see the big, linked-up picture. Her modestly sized, elaborate and detailed construction does not illustrate an autopsy, rather its making is framed by Gelb's three principles. Her artwork invites us to read meaning into the conjunction of objects. Faced with an image as powerful as that of a brain removed from its bony container, we can take up her invitation.

But why the walnut? It clearly exploits the visual resonance between a furrowed walnut plucked whole from its halved shell and the wrinkled configuration of the brain. It also refers to the ancient and cross-cultural idea of the microcosm and macrocosm, which highlights similarities of form and function across every scale in nature and the wider Universe. Old herbal medicine in both Western and Eastern cultures used this doctrine to help determine the source of treatments. A herb or fruit that resembles a human organ was seen as potentially efficacious for treating a disease of that organ.

Before we smile patronizingly at such ancient mysticism, it is curious to note that walnuts could have an effect on some ageing disorders of the brain. The late James Joseph and his team at Tufts University in Boston, Massachusetts, reported in the British Journal of Nutrition in 2009 that a diet including walnuts seemed to improve cognitive function in ageing rats.

As happens in the best scientifically orientated artworks, a visual starting point opens up a range of associations across historical and contemporary practice.

Martin Kemp is emeritus professor of the history of art at the University of Oxford, UK. 